[CONTRIBUTION FROM THE LABORATORIES OF THE MASSACHUSETTS INSTITUTE OF TECHNOLOGY.]

\title{
LECTURE EXPERIMENTS ILLUSTRATING THE ELECTRO- LYTIC DISSOCIATION THEORY AND THE LAWS OF THE VELOCITY AND EQUILIBRIUM OF CHEMICAL CHANGE.
}

\author{
By A. A. NoYes AND A. A. BlaxchaRD. \\ Received September 20 , rgoc.
}

INTRODUCTION.

\section{T}

$\mathrm{HE}$ experiments to be described in this paper were originally devised as an accompaniment to an extended course of lectures on theoretical chemistry. ${ }^{1}$ Most of the principles illustrated are, however, of such fundamental importance that they must soon be generally introduced even into elementary lecture courses on inorganic and analytical chemistry; and it is the hope of the authors that this series of experiments will not only be of assistance to teachers of theoretical chemistry, but will also serve in some degree to hasten and facilitate the introduction of instruction in the principles of chemical equilibrium and of electrolytic dissociation into the elementary courses.

A few lecture experiments of a somewhat similar character have been previously published. Those of Kiister, ${ }^{2}$ and of Lash Miller and Kenrick, ${ }^{3}$ may be specially referred to.

The experiments are presented below in the form believed to be most convenient for those desiring actually to perform them. They are described in great detail, with the hope of saving others the large amount of time which has been expended in the attempt to find the best conditions for rapid and effective lecture demonstrations. It is, nevertheless, true that a slight accidental variation in one of the conditions, such as the dimensions of the apparatus employed, the temperature, or the concentration of the substances, may make modifications necessary; it is, therefore, of course, advisable to try the experiments before the lecture.

1 Many of these experiments were shown in a lecture on "Some Applications of the Electrolytic Dissociation Theory" given before the American Chemical Society at its Columbus Meeting.

2 Ztschr. Electrochem, , 4, 105

3 This Journal, 22, 291-300; See also Jones and Allen: Am. Chem.J., I8, 377 ; and Stieg. litz: Ibid., 23, 404 . 
The principles illustrated have been stated as briefly as possible in a non-mathematical form: in spite of the familiarity of these principles, it has seemed desirable to state them explicitly, in order to make clear the exact significance of each experiment.

\section{PART I -ELECTROLYTIC DISSOCIATION.}

\section{EXPERIMENT I}

Principle Illustrated. -The physical properties of dilute aqueous salt solutions are adclitive, being dependent on the nature of the metal and of the acid component of the salt, but not on their mutual relations. Since most salts in moderately dilute aqueous solution are, according to the dissociation theory, almost completely dissociated into their ions, all properties of such solutions must be the resultant of the properties of the solvent and of the separate ions, each ion having a definite influence which is independent of the nature of the other ion simultaneously present and of that of the undissociated salt from which it originated. This principle is best illustrated in the case where one of the ions has a characteristic property, such as color, not possessed at all by the other ion. Thus, all the salts of a metal whose ion is colored must have the same color in dilute aqueous solution, provided the negative ion of the salt is colorless, however different may be the colors of the undissociated salts. The colors of the latter may be shown, either in the solid state, or in a concentrated alcoholic solution.

Preparation of the Experiment.-Dissolve 60 grams of powdered $\mathrm{CoCl}_{2}, 6 \mathrm{H}_{2} \mathrm{O}$ and 73 grams of $\mathrm{Co}\left(\mathrm{NO}_{3}\right)_{2} \cdot 6 \mathrm{H}_{2} \mathrm{O}$ each in $100 \mathrm{cc}$. of alcohol, thus making a fivefold normal solution of each salt. Dissolve 27 grams of anhydrous cupric chloride (made by heating the blue crystals in a porcelain dish over a free flame) and $4^{8}$ grams of $\mathrm{Cu}\left(\mathrm{NO}_{3}\right)_{2} \cdot 3 \mathrm{H}_{2} \mathrm{O}$, each in $100 \mathrm{cc}$. of absolute alcohol, thus making a fourfold normal solution of each salt. Pass into the cupric chloride solution a few cubic centimeters of dry hydrochloric acid gas. Place $20 \mathrm{cc}$. of each of these solutions in four large test-tubes. Provide also distilled water, concentrated ammonia, and $20 \mathrm{cc}$. of concentrated potassium cyanide solution.

Experiment.-Add $100 \mathrm{cc}$. of cold water to each of the four solutions. Then add $20 \mathrm{cc}$. of ammonia to one of the copper 
solutions, and $20 \mathrm{cc}$. of concentrated potassium cyanide solution to one of the cobalt solutions.

Observations. - The alcoholic solutions of cobalt chloride and nitrate are deep violet-blue and purplish red, respectively, but become of the same shade of pink after dilution with water. Those of copper chloride and nitrate are originally greenish black and light blue, respectively, but beccme of the same shade of blue on dilution. The ammonia added to the copper solution produces a much more intense blue coloration of a different shade, and the potassium cyanide added to the cobalt solution changes its color from pink to brown.

Remarks. - The hydrochloric acid gas is passed into the cupric chloride solution in order to avoid the precipitation of a basic salt on subsequent dilution.

The change in color accompanying the dilution may well be due mainly to hydration of the salts rather than to dissociation of them; but whatever the cause, the experiment evidently still illustrates the principle of the dependence of the properties of dilute aqueous solutions on those of the separate ions. The addition of aumonia to the copper solution transforms the copper ions into complex positive ions of the form $\mathrm{Cu}\left(\mathrm{NH}_{3}\right)_{x}$; and that of potassium cyanide to the cobalt solution converts the cobalt ions into complex negative ions, probably of the composition, $\mathrm{Co}(\mathrm{CN})_{6}$. These explanations show that the exceptions to the principle of additivity which are illustrated by the last part of the experiment are in full accord with the dissociation theory; for the nature of the ions having changed, the color of the solution must change likewise.

EXPERIMENT II.

Principle Illustrated.-Metathetical reactions between dissolved salts take place instantaneously, whatever may be the nature of the salts, while those in which organic compounds are involved commonly require an appreciable time which varies greatly with the nature of the compound. This difference of behavior is, according to the dissociation theory, due to the fact that the ions of salts, being already separated by the solvent, are capable of immediate combination, when those which have the tendency to combine are brought into contact, while the component elements 
or radicals of organic substances must be separated from each other before they can enter into a metathesis. A very slight degree of dissociation of the salts is sufficient, however, owing to the fact that the ions removed by the metathesis are immediately replaced by others by the spontaneous and almost instantaneous process of dissociation.

Preparation.-Prepare roo cc. of nearly saturated solution of potassium bromide and of zinc bromide in alcohol, and equal quantities of 5 per cent. solutions of isopropyl bromide, ethyl bromide, and phenyl bromide. Place these solutions in five lecture jars. Prepare $150 \mathrm{cc}$. of a nearly saturated alcoholic solution of silver nitrate (about 25 grams to the liter). Provide a stirring rod with one end flattened at right angles to the rod, for vertical stirring.

Experiment.-Add $20 \mathrm{cc}$. of the alcoholic silver nitrate solution to each of the five bromide solutions and stir.

Observations. - The potassium and zinc bromide solutions both give instantly with the silver nitrate a copious flocculent precipitate. The isopropyl bromide solution becomes turbid immediately, but an appreciable time is seen to be required for the completion of the precipitation. The ethyl bromide remains clear for a few seconds, and then becomes turbid, but the turbidity increases very slowly on standing. The phenyl bromide gives no turbidity, even after long standing.

Remarks.-Care must be taken that the ethyl and phenyl bromides are free from hydrobromic acid and bromine. These impurities, if present, may be removed by washing in a separatory funnel, first with dilute sodium carbonate solution, and then with water.

\section{EXPERIMENT III.}

Principle Illustrated. - The passage of electricity through a salt solution causes the metallic component of the salt to move in the direction of the current, and the acid component to move in the opposite direction. The products into which salts in aqueous solution are dissociated are therefore electrically charged molecules or ions.

Preparation.-Cut ro grams of agar-agar in small pieces, place in a casserole, add $500 \mathrm{cc}$. of cold water, heat to boiling, 
and boil until a clear solution is formed. Strain while still hot through a piece of cloth, and preserve the jelly formed on cooling as a stock for use in this and the next two experiments. To $35 \mathrm{cc}$. of the remelted agar add $8 \mathrm{cc}$. of saturated cupric chloride solution, a few drops of acetic acid, and $6 \mathrm{cc}$. of saturated potassium bichromate solution. Pour enough of this solution into a $U$-tube $2 \mathrm{~cm}$. in diameter and $16 \mathrm{~cm}$. high to fill the tube $3-4 \mathrm{~cm}$. above the bend, and allow the jelly to harden. Sprinkle on each surface a little bone-black to mark the boundary, then pour into each arm a few drops (to fix the bone-black in place) of a solution made by mixing $4 \mathrm{cc}$. of saturated potassium chloride solution with $15 \mathrm{cc}$. of the melted stock-agar, cool in water, and after a few moments pour in enough more of this mixture to form a layer a few centimeters high in each arm. After this has hardened, pour into each arm a few cubic centimeters of saturated potassium chloride solution, insert electrodes of platinum wire in both arms, and connect them through an open switch and a 32 candle-power iro-volt lamp with the terminals of a I Io-volt direct-current circuit. Place the $U$-tube in a beaker of water containing a large quantity of ice.

Experiment.-Close the switch and allow the current to pass through the tube for ten to thirty minutes, stopping it temporarily if the agar should show signs of melting.

Observations. - The copper chromate jelly filling the bottom of the tube has a deep green color. After the current has passed for some minutes a blue zone (due to the copperions) appears above the bone-black boundary in one arm, and a yellow zone (due to chromate ions) above the bone-black in the other arm, and these zones both extend upwards as the electrolysis continues. The zones immediately below the bone-black in both arms also acquire a blue and yellow color respectively, while the central portion beneath remains unchanged in color.

Remarks. - The experiment should be prepared not more than an hour or two before the lecture, as otherwise the boundaries will not be sharp, owing to diffusion. It is desirable to use, as directed, a larger proportion of copper salt than corresponds to the normal chromate, since otherwise the color of the blue copper zone will not be deep enough to be visible at a distance. The 
acetic acid is added to prevent the precipitation of a basic chromate. The potassium chloride in the arms of the tube serves to form a colorless conducting layer, its ions carrying the current to and from the boundary of the copper chromate. Accurate experiments have shown that the addition of enough agar to form a solid jelly does not affect the rate of migration of the ions more than a few per cent.; there is, therefore, no objection to its use for purposes of demonstration.

\section{EXPERIMENT IV.}

Principle Illustrated. -The rate of migration of the ions is dependent on their nature. The hydrogen ion moves most rapidly, the hydroxyl ion about one-half as fast, and other inorganic ions from one-fourth to one-eighth as fast as the hydrogen ion. The rate of motion of the ions and the number of them present are the two factors determining the conductivity of a solution.

Preparation. $-F$ ill the bend of a U-tube $(2 \mathrm{~cm}$. in diameter, and $16 \mathrm{~cm}$. high) and one of its arms up to a point $5 \mathrm{~cm}$. from the top with a jelly made of $16 \mathrm{cc}$. of saturated potassium chloride solution, 12 drops of phenolphthalein solution, $50 \mathrm{cc}$. of the stock-agar (see Expt. III), and just enough potassium hydroxide to color it deeply red. Fill the other arm of the tube up to 5 $\mathrm{cm}$. from the top with some of the same jelly to which has been added just twice as much hydrochloric acid as is needed to decolorize it. Place platinum wires in the air spaces at the top of each arm, and connect these wires through a 32-candle-power i Iovolt lamp with the terminals of a I lo-volt direct-current circuit in such a manner that the current will enter the arm containing the colored jelly. Place the tube in a beaker of water containing pieces of ice. Prepare a mixture of $0.5 \mathrm{cc}$. of hydrochloric acid (sp. gr. I.I2), $6 \mathrm{cc}$. of saturated copper chloride, and $20 \mathrm{cc}$. of water, and a mixture of $2 \mathrm{cc}$. of Io per cent. potassium hydroxide solution with $20 \mathrm{cc}$. of saturated potassium chloride solution.

Experiment.- Nearly fill the arm of the tube containing the colored jelly with the solution of copper chloride and hydrochloric acid and the other arm with the solution of potassium hydroxide 
and chloride, close the switch, and allow the current (of about 0.5 ampere) to pass for about fifteen minutes.

Observations. - A colorless zone (due to the hydrogen ions) descends into the pink jelly to a depth of about $5 \frac{1}{2} \mathrm{~cm}$, , and this is followed by a blue zone (due to the copper ions) to a depth of I cm. In the other arm a pink zone (due to the hydroxyl ions) descends into the colorless jelly to a depth of about $2 \frac{1}{2} \mathrm{~cm}$.

\section{EXPERIMEN'T V.}

Principle Illustrated.-The composition of the ions can be determined by migration experiments. Thus in the case of the double cyanide of potassium and silver, the silver can be shown to move against the current, thus proving that it forms a part of the negative ion, which has in fact been shown to have the composition $\mathrm{Ag}(\mathrm{CN})_{2}$.

Preparation.-Charge a U-tube, $2 \mathrm{~cm}$. in diameter and $16 \mathrm{~cm}$. high, not more than an hour or two before the experiment is to be performed, in the manner shown in the accompanying scale

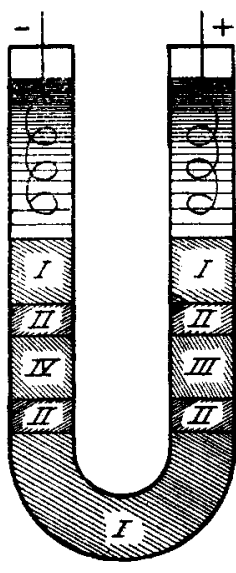

Fig. I. drawing, with various jellies, designated I, II, III, and IV. Jelly No. I, is prepared from $30 \mathrm{cc}$. of the stock-agar (Expt. No. III), $6 \mathrm{cc}$. of saturated potassium nitrate solution, and 6 cc. of a suspension of washed precipitated zinc sulphide in water; jelly No. II, from $5 \mathrm{cc}$. of the agar and $4 \mathrm{cc}$. of saturated potassium nitrate solution; jelly No. III, from $5 \mathrm{cc}$. of the agar, I cc. of saturated potassium nitrate, and I cc. of a 3 per cent. silver nitrate solution; and jelly No. IV, from $5 \mathrm{cc}$. of the agar, I cc. of saturated potassium nitrate solution, and I cc. of a potassium silver cyanide solution made by adding concentrated potassium cyanide solution, drop by drop, to a 3 per cent. silver nitrate solution until the precipitate first formed redissolves. In charging the tube, pour in each portion of melted jelly through a long-necked funnel to avoid contaminating succeeding portions, and cause each to harden by immersing in cold water before adding the next. Make each of the layers of a thickness corresponding to that shown in the drawing, taking especial care that the layers 
of jelly II be not more than I cm. thick, since otherwise too long a time will be required for the experiment; and in forming the last layers, pour in first a few drops of the solution, allow this to solidify, and then add the remainder, in order to prevent them from taking up and mixing with any of the jelly beneath. Pour into each arm on top of the jelly some saturated potassium nitrate solution, insert platinum wires, and connect them with the terminals of a r ro-volt direct-current circuit in such a manner that the current will enter the arm containing the silver nitrate jelly (No. III). Immerse the tube in a beaker of ice and water.

Experiment.-Cause the current (which will be one of about 0.35 ampere) to pass through the tube for fifteen to twenty minutes, stopping it temporarily or inserting additional resistance if the agar shows signs of melting.

Observations. - The zinc sulphide jelly at the bottom, which is pure white at first, after a few minutes begins to blacken at its surface below the silver nitrate, and a few minutes later at its other surface below the potassium silver cyanide, the blackening extending rapidly downward on both sides. The zinc sulphide layers near the top of the arms remain white throughout the experiment.

Remark.-Zinc sulphide is used instead of an alkaline sulphide to indicate the presence of silver, since, being nearly insoluble, it does not move appreciably with the current.

\section{EXPERIMENT VI.}

Principle Illustrated.-The molecular conductivity of salts increases on dilution at first rapidly, and then more slowly, and finally attains a constant maximum value which is not affected by further dilution. The molecular conductivity of a dissolved substance is the conductivity of that volume of its solution which contains I mol of the substance, when the solution is placed between parallel electrodes of sufficient extent, I $\mathrm{cm}$. apart. According to the dissociation theory, the change of this property on dilution results from the fact that electricity is transported through the solution solely by the ions, and the number of these is increased at the expense of the undissociated portion of the salt by increased dilution, up to the point where the salt is com- 
pletely dissociated, after which further dilution has, of course, no further effect.

Preparation.-Construct a high, narrow trough with parallel glass sides and wooden ends and base in the following manner : Cover one of the broad surfaces of each of two strips of wood 45 $\mathrm{cm}$. long, I $\mathrm{cm}$. thick, and $2.2 \mathrm{~cm}$. wide, previously oiled and shellaced, with a strip of thin silver foil, attaching this to the wood by means of shellac. Insert these two strips of wood with the silver-covered surfaces towards each other and uniformly i 8.5 $\mathrm{cm}$. apart between two sheets of plate glass $(45 \times 22.5 \times 0.6 \mathrm{~cm}$.) so as to leave a free space about I $\mathrm{cm}$. deep outside of the strips. Clamp the apparatus together temporarily, and fill this free space with a melted cement made by stirring one part of beeswax into five parts of melted rosin. Bind tightly over each of these two ends a piece of stout cloth or canvas, and attach it to the glass sides by means of shellac, so as to hold the whole in position. Insert this cell into a wooden base so made that the cell will fit tightly into a groove to a depth of $\mathrm{I} .5 \mathrm{~cm}$.; pour on to the bottom inside more of the above cement heated enough to be very fluid, and allow the cement to cool, so as to form an even horizontal layer. Solder wires to the tops of the silver electrodes. Connect the resistance cell thus constructed in series with a storage cell, a switch, and a suitable current indicator. Use as a current-indicator any Weston station voltmeter, from which the series resistance coil in the back has been removed or short circuited. (The moving coils in all the Weston station voltmeters have a resistance of about 75 ohms; and a full scale deflection is obtained with a current of about 0.0 I 2 ampere; the station type is used since it has a pointer which can easily be seen at a distance.) Prepare a stirring rod by closing up one end of a wide glass tube 0.6 meter long. Provide a glass siphon suitable for emptying the resistance cell, and fill it with water; also a funnel attached to a tube long enough to reach the bottom of the cell. Measure out in four graduates three portions of distilled water of 60,240 , and $960 \mathrm{cc}$, respectively. Prepare 20 $\mathrm{cc}$, of a fourfold normal silver nitrate solution by dissolving 3.5 grams of solid silver nitrate in water, and making the volume up to $20 \mathrm{cc}$. 
Experiment.-Fill the resistance cell with distilled water, and close the switch for a few moments. Insert the siphon, and empty the cell completely, tilting it at the end. Then pour into the bottom of the cell through the funnel the $20 \mathrm{cc}$. of fourfold normal silver nitrate solution. Close the switch, and note on the board the scale reading. Then pour into the cell the $60 \mathrm{cc}$. portion of water, stir, and note the reading. Then pour in successively the $240 \mathrm{cc}$. and the $960 \mathrm{cc}$. portions of water, noting the readings after the complete addition of each portion.

Observations.-A scarcely noticeable deflection of the needle is produced when the cell is filled with pure water. The scale-readings corresponding to the most concentrated solution and the first fourfold dilution of it are found to differ greatly, those of the first and second dilutions much less, and those of the second and third dilutions by only a relatively small amount; thus the scale-readings in an actual experiment were $7.1,8.4,9.2$, and 9.5 .

Remarks.-In the appatatus and experiment above described the distance between the electrodes, though not $I \mathrm{~cm}$., is a constant distance, and the amount of the salt used, though not I $\mathrm{mol}$, is a definite amount, so that a satisfactory illustration is given of the property of molecular conductivity and of its variation with the dilution, a phenomenon, which, as is well known, was the starting-point of the electrolytic dissociation theory, and one which forms the basis of the most important method of determining dissociation.

The molecular conductivities of the silver nitrate solutions are at the four concentrations, according to Kohlrausch, approximately $39,63,80$, and 92 units. The effect of having the resistance (about $75 \mathrm{ohms}$ ) of the voltmeter constantly in series with the cell is to make the apparent change of conductivity less than the true change; nevertheless, the effect is sufficiently great to make the demonstration an entirely satisfactory one, as is shown by the scale-readings cited above.

If copper electrodes are substituted for the silver ones, and a copper salt solution for the silver nitrate, the change of conductivity with the dilution is still greater; but the experiment is far less satisfactory, for it is not possible, owing to the great dilu- 
tion required, to illustrate the approach to a maximum value. EXPERIMENT VII.

Principle Illustrated.-Different acids of the same concentration differ greatly in their conductivities and degrees of dissociation; but their neutral salts have approximately the same conductivities and same degrees of dissociation.

Preparation.-Fill four $500 \mathrm{cc}$. bottles with half-normal solutions of hydrochloric acid, sulphuric acid, chloracetic acid, and acetic acid, prepared by weighing out the calculated amounts of the commercial acids. (It is desirable, however, to prove the approximate equivalence in the concentrations of the four acids by titration with normal potassium hydroxide, and to adjust them if they differ by more than five per cent.) Distinctly label these solutions, and reserve them for use in this and later experiments.

Make the apparatus represented in the accompanying figure in the following manner: Procure four glass tubes as nearly alike as possible, of an internal diameter of about $3 \mathrm{~cm}$. and a length of $20 \mathrm{~cm}$. (The straight parts of "student lamp" chimneys can be used for the purpose.) Place in one end of each of these tubes a rubber stopper with one hole in which has been inserted a sinall glass tube containing a stout copper wire to which a thin circular platinum disk is soldered, this disk covering the small end of the rubber stopper and being attached to it by means of sealing-wax. Set up the tubes in a vertical position with the stoppers at the bottom, holding the tubes in place by means of clamps or by a suitable wooden frame made for the purpose. Insert in the upper end of each of the tubes a rubber stopper carrying a small thick-walled glass tube $22 \mathrm{~cm}$. long through which passes a stout copper wire, to the lower end of which is soldered, at right angles to the wire, a thin circular platinum disk of a diameter a few millimeters less than that of the tube, the disk being reinforced by a thick layer of sealing-wax on its upper surface. These tubes should move freely through the stoppers, but yet be held in place by them. Connect the wire issuing from the bottom of each large tube with a 32 -candlepower I Io-volt lamp placed in a socket beneath; make also the other connections shown in the drawing, using short coils of 
covered flexible wires between the four upper electrodes; and finally, connect through an open switch the upper electrodes with one terminal and the lamps with the other terminal of a

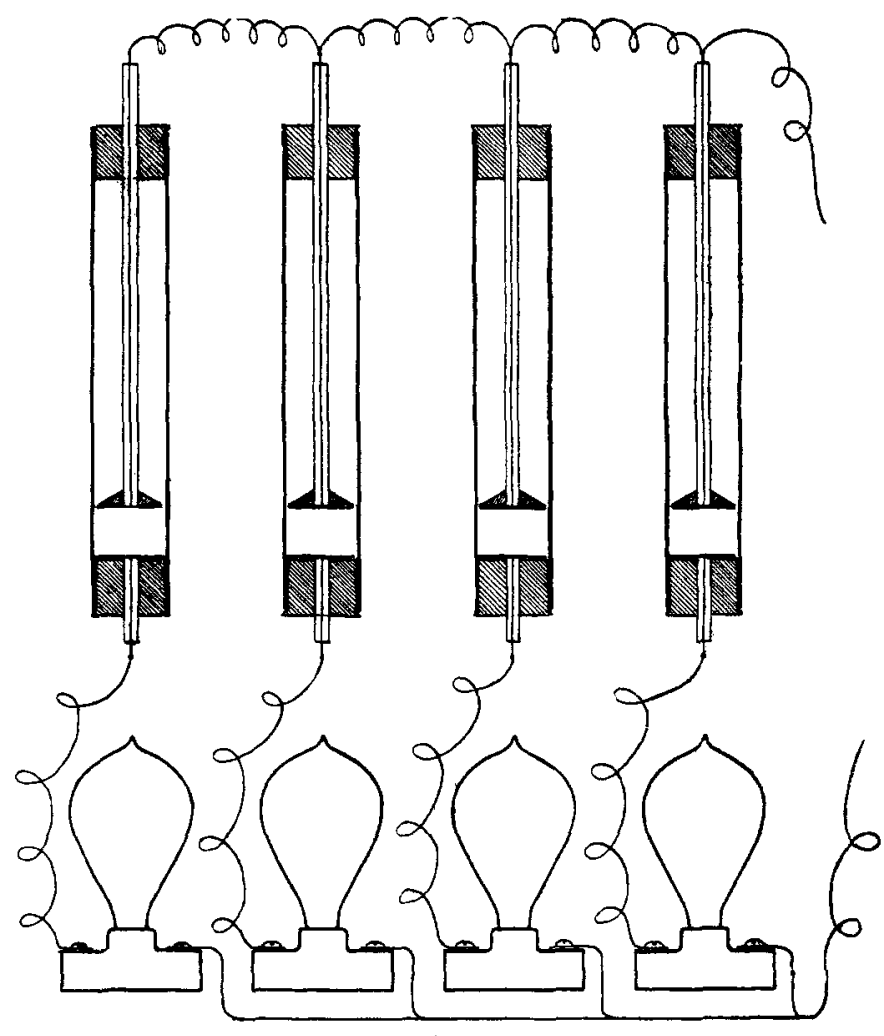

jiig. 2.

I IO-volt alternating circuit. Place in each tube $I 20 \mathrm{cc}$. of distilled water and set the electrodes one-third of the distance from the bottom. Provide a $5 \mathrm{cc}$. graduated pipette, a little phenolphthalein solution, and $50 \mathrm{cc}$. of normal potassium hydroxide solution.

Experiment.-Raise the stoppers carrying the upper electrodes, and add to each of the four tubes with the pipette $5 \mathrm{cc}$. of each of the four half-normal acids, so that they will be in the order, acetic, chloracetic, sulphuric, hydrochloric, and stir thoroughly. Re-insert the electrodes, darken the room somewhat, and close 
the circuit. Raise the electrode in the hydrochloric acid solution to the top of the tube, and then adjust the heights of the other three electrodes so that all the lamps beneath glow with equal brilliancy. Admit the light to the room, and call attention to the relative heights of the four electrodes. Then raise the stoppers, and add to each tube two or three drops of phenolphthalein solution and from the pipette about $2.5 \mathrm{cc}$. of a normal solution of pure potassium hydroxide, adding the last few drops in each case slowly and with vigorous stirring, until the solution remains permanently colored. Again darken the room, set the electrode in the potassium chloride solution about one-third of the total distance from the bottom, and adjust the other three electrodes so that all four lamps glow with equal brilliancy. Admit the light to the room, and call attention to the heights of the four electrodes.

Observations. - Starting as directed with the four electrodes at the same height, about one-third of the distance from the bottom, it is seen when the circuit is closed that the lamps are more brilliant in correspondence with the order, hydrochloric, sulphuric, chloracetic, acetic, the lamp beneath the last-named acid not glowing perceptibly. After the lamps are brought to equal brilliancy by adjusting the electrodes, it is seen that if the upper electrode in the hydrochloric acid is at the top, that in the sulphuric acid is about one-fourth of the distance down, that in the chloracetic acid three-fourths of the distance down, and that in the acetic acid nearly touching the lower electrode. It is seen that nearly equal amounts of potassium hydroxide are required for the neutralization of all four acids, thus proving them of equal concentration. Finally after adjusting the lamps to constant brilliancy, the electrodes in the salt solutions are seen to stand at not far from the same heights.

Remarks. - The chloracetic acid solution must be prepared not more than a few days before it is used, since, otherwise, it will contain an appreciable amount of hydrochloric acid. If an alternating circuit is not available, a direct circuit may be used, but in that case it is desirable that the upper electrodes be made convex downwards so that the gas will escape, and that the circuit be closed only for as short a time as is necessary. 
Instead of charging the tubes of the conductivity apparatus during the lecture with the acids, this may be done beforehand, in order to save time; but in that case, the audience is obliged to take for granted the fact that the acids used in the later experiments are identical with those used in this one, a fact which is made obvious if the portions of acids are in each case drawn from the same bottles. In fact, throughout most of the following experiments, much time can be saved in the lecture by measuring out the solutions in advance, the audience being asked to accept the lecturer's statement in regard to their composition. While the purpose of such experiments is to illustrate rather than to demonstrate, it is nevertheless true that they become less satisfactory, the more the audience has to take for granted. To what extent it is desirable to carry the preparation of the experiments, will therefore depend on the character of the lectures, on the time that can be given to experiments, and whether the services of an assistant are available during the lecture.

Lecturers will, we believe, find themselves repaid for the small amount of labor involved in the construction of the apparatus just described; for not only is the above experiment a very satisfactory one, but the same apparatus can be employed for the demonstration of Ostwald's dilution law (see Expt. XII), and for the illustration of the conductivity and dissociation of many substances besides those here used. It should be stated that the apparatus was devised and first constructed by Dr. W. R. Whitney of our Institute.

\section{PART II.-THE VELOCITY OF CHEMICAL REACTIONS.}

\section{EXPERIMENT VIII.}

Principle Illustrated. -The rate at which a chemical change takes place is proportional to the concentration of each of the substances taking part in it. ${ }^{1}$

Preparation. - In a 2-1iter bottle put I600 cc. of distilled water, $50 \mathrm{cc}$. of half-normal hydrochloric acid, and $40 \mathrm{ce}$. of starch solution (made by rubbing I gram of potato starch to a paste with

I As is well known, the rate is in some cases proportional to that power of the concentration which corresponds to the number of molecules involved; but this experiment illustrates only the simpler case. 
$5 \mathrm{cc}$. of cold water, pouring $\mathrm{r}_{50} \mathrm{cc}$. of boiling water over it, allowing the undissolved part to settle, and decanting the supernatant liquid). Prepare a 0.5 normal solution of potassium bromate by dissolving 7 grams of the salt in half a liter of water; also a 0.5 normal solution of potassium iodide by dissolving 42 grams of it in half a liter of water. Reserve these solutions for use in the three following experiments: Provide four $500 \mathrm{cc}$. white glass stoppered bottles, $8 \mathrm{~cm}$. in diameter, and set two ro cc. graduates in front of each of them. Prepare a standard blue solution in a fifth $500 \mathrm{cc}$. bottle by adding to $400 \mathrm{cc}$. of water, Io cc. of the starch solution and I cc. of a solution of I gram of iodine and 2 grams of potassium iodide in $500 \mathrm{cc}$. of water. Place all five bottles on the lecture table against a background of white paper. Provide a large clock (or stop-watch) with a second hand; also a $500 \mathrm{cc}$. graduate.

Experiment.-Pour into each of the four bottles from the large graduate $400 \mathrm{cc}$. of the acid solution in the 2 -liter bottle. Pour into the four pairs of $\mathrm{IO} \mathrm{cc}$. graduates respectively $5 \mathrm{cc}$. of the bromate and $5 \mathrm{cc}$. of the iodide solution, Io $\mathrm{cc}$. bromate and $5 \mathrm{cc}$. iodide, $5 \mathrm{cc}$. bromate and Io $\mathrm{cc}$. iodide, and Io $\mathrm{cc}$. bromate and Io cc. iodide. Add to each of the four bottles the bromate solution from the graduate before it, and then, at a definite instant, when the second hand of the clock shows an even minute. add simultaneously to the four bottles the measured portions of iodide solution, quickly insert the stoppers, and shake vigorously. Note on the board by reference to the clock the times when each of the solutions becomes of the same shade of blue as the standard, placing the latter in succession by the side of the different reaction bottles.

Observations. - The mixture containing the Io $\mathrm{cc}$. of both bromate and iodide will become of the same shade of blue as the standard in about thirty seconds. The mixtures with Io cc. bromate and $5 \mathrm{cc}$. iodide and with $5 \mathrm{cc}$. bromate and ro cc. iodide will both require the same interval of time, which will be about twice as great as that for the first mixture. The mixture containing $5 \mathrm{cc}$. of both bromate and iodide will require nearly twice as much time as these last mixtures. 
EXPERIMEN'T IX.

Principle Illustrated.-Equal increments of temperature cause an equal multiplication of the velocity of any definite chemical reaction. ${ }^{1}$

Preparation.-Mix in a 2-liter bottle I I00 cc. of water, 100 cc. of 0.5 normal hydrochloric acid, and $30 \mathrm{cc}$. of starch solution. Put $400 \mathrm{cc}$. of this solution into each of three $500 \mathrm{cc}$. white glass-stoppered bottles ( $8 \mathrm{~cm}$. in diameter); bring by means of suitable baths one of these solutions to $4^{\circ}$, another to $16^{\circ}$, and the third to $28^{\circ}$; and allow the bottles to stand in the baths until the experiment is to be shown. Mix in a $50 \mathrm{cc}$. graduate Io cc. of 0.5 normal potassium bromate, Io cc. of 0.5 normal potassium iodide, and $25 \mathrm{cc}$. of water, all measured as closely as possible; and pour just Io $\mathrm{cc}$. of this mixture into each of three Io cc. graduates. Provide a clock with a second hand, and a large thermometer of short range whose readings are visible at a distance. Prepare in a fourth bottle, just as in Expt. VIII, a blue iodide of starch solution to serve as a standard. Place all four bottles against a background of white paper.

Experiment. - Insert the thermometer in succession in each of the three bottles, and note the temperatures on the board. At a definite instant, when the clock shows an even minute or half minute, pour the three portions of bromate solution into the three bottles, stopper quickly, and shake vigorously. Note on the board the times at which the three solutions reach the same depth of blue as the standard.

Observations. - The times required to reach the color of the standard will be about thirty-two seconds, fifty-eight seconds, and one hundred and five seconds at $28^{\circ}, \mathrm{I} 6^{\circ}$, and $4^{\circ}$, respectively.

Remarks.-The hottest solution must not be above $30^{\circ}$, since otherwise a considerable error arises from the lessening of the depth of color of the iodide of starch. Between $4^{\circ}$ and $30^{\circ}$, however, the intensity of color undergoes a scarcely perceptible change, as was proved by a special experiment.

EXPERIMENT $\mathrm{X}$.

Principle Illustrated. - The effect of acids in accelerating chem-

1 This principle may be somewhat more explicitly stated as follows: In the case of any chemical reaction, the velocity-coefficients corresponding to an arithmetical series of temperatures, form a geometrical series.

23-22 
ical reactions is roughly proportional to their electrical conductivities. The effect is primarily dependent on the hydrogen ions, and is in most cases approximately proportional to their concentration. ${ }^{1}$

Preparation.-Mix in a 2 liter bottle, $40 \mathrm{cc}$. of 0.5 normal potassium iodide, $40 \mathrm{cc}$. of 0.5 normal potassium bromate, $40 \mathrm{cc}$. of starch solution, and $\mathrm{I} 600 \mathrm{cc}$. of water. Place on the lecture table the four half-normal solutions used in Expt. VII, four 500 cc. bottles, four Iocc. graduates, a $500 \mathrm{cc}$. graduate, and white paper arranged so as to serve as a background.

Experiment.-Measure with the large graduate $400 \mathrm{cc}$. of the bromate-iodide mixture into each of the four bottles. Pour into the small graduates just ro cc. of each of the half-normal acids, and place them in front of the four bottles in the order hydrochloric, sulphuric, chloracetic, acetic acid. Add at the same instant the contents of the four graduates to the bottles, stopper quickly, and shake.

Observations. - The solution containing the hydrochloric acid becomes deep blue almost immediately; that with the sulphuric acid, within half a minute; that with the chloracetic acid, after three or four minutes; and that with acetic acid, only after three or four hours.

\section{EXPERIMENT XI.}

Principle Illustrated.-Neutral substances sometimes act as powerful catalytic agents.

Preparation.-Place in a liter bottle $800 \mathrm{cc}$. of water, $40 \mathrm{cc}$. of 0.5 normal potassium iodicle, $40 \mathrm{cc}$. of 0.5 normal potassium bromate, and $20 \mathrm{cc}$. of starch solution. Provide a half-normal solution of acetic acid and a neutral saturated solution of ferrous sulphate; also, a graduated I cc. pipette, two $500 \mathrm{cc}$. bottles, and two 50 $\mathrm{cc}$. and one $500 \mathrm{cc}$. graduates.

Experiment.-Measure into each of the two bottles, with the large graduate, $400 \mathrm{cc}$. of the bromate-iodide mixture. Measure out in each of the two graduates $50 \mathrm{cc}$. of the half-normal acetic acid, and add to one of the graduates $0.5 \mathrm{cc}$. of the ferrous sul-

${ }^{1}$ In the case of the bromate-iodide reaction the accelerating effect increases much more rapidly than the concentration of the hydrogen ions (see Zischr. phys. Chem., Ig, 599); but in spite of this exceptional behavior, it can be used to demonstrate the order of the effect of different acids, which is all that is attempted in this experiment. 
phate solution. At the same moment add the contents of the graduates to the two bottles, quickly insert the stoppers, and shake.

Observations.-The solution containing the ferrous sulphate becomes deep blue within a few seconds, while that not containing it requires as many minutes to reach the same depth of color.

\section{PART III.--CHEMICAL EQUILIBRIUM OF DISSO- CIATED SUBSTANCES.}

\section{EXPERIMENT XII.}

Principle Illustrated.--The molecular conductivity, and therefore the dissociation, of a slightly dissociated acid varies as the square root of the volume in which a definite quantity of the acid is contained, in accordance with the requirement of the mass action law. The general expression of the effect of dilution on the dissociation of acids, or of the so-called Ostwald's dilution law, is $\frac{d^{2}}{(\mathrm{I}--\bar{d}) v}=\mathrm{K}$, where $d$ is the degree of dissociation, $v$ the volume containing I molecule, and $\mathrm{K}$ the dissociation-constant. If $d$ is small, the quantity ( $I-d)$ can be considered equal to unity without great error, whence the above-stated principle follows.

Preparation.--Place on the lecture table the conductivity apparatus and the half-normal chloracetic acid solution used in Expt. VII. Provide two $300 \mathrm{cc}$. beakers and a $50 \mathrm{cc}$. and a $200 \mathrm{cc}$. graduate, also half a liter of distilled water and have this at the same temperature (that of the room) as the chloracetic acid. Connect the conductivity apparatus through a switch with the terminals of a i ro-volt alternating circuit.

Experiment.--Mix in a beaker $50 \mathrm{cc}$. of the half-normal chloracetic acid solution with $150 \mathrm{cc}$. of water. In a second beaker dilute $50 \mathrm{cc}$. of the solution so obtained with $150 \mathrm{cc}$. of water. Nearly fill three of the tubes of the conductivity apparatus with the half-normal chloracetic acid from the bottle, and with the two diluted solutions in the beakers, respectively. Raise the electrode in the tube containing the most concentrated acid to the top, darken the room, close the circuit, and adjust the other two electrodes so that the three lamps beneath glow with equal brilliancy. 
Open the circuit, admit the light, and call attention to the position of the electrodes.

Observations.-At the close of the experiment it is seen that the electrodes in the half-normal solution are about twice as far apart as those in the eighth-normal solution, and about four times as far apart as those in the $1 / 32$-normal.

Remarks. - The remark on Expt. VII in regard to the use of a direct current applies here also.

The distances between the electrodes are directly proportional to the specific conductivities of the solutions; consequently, the molecular conductivities are proportional to the products of these distances into the volumes in which a definite amount of acid is contained.

Chloracetic acid is $5.4,10.5$, and 20.0 per cent. dissociated in $\mathrm{I} / 2, \mathrm{I} / 8$, and $\mathrm{I} / 32$-normal solutions respectively. Owing to the rather large degree of dissociation, the proportionality between this and the square root of the volume is not exact; but the deviations are not great enough to be noticeable in the experiment here described.

\section{EXPERIMENT XIII.}

Principle Illustrated.--The dissociation of a slightly dissociated acid (or base) is very greatly reduced by the addition of an equivalent quantity of one of its neutral salts. For, according to the mass action law, the product of the concentrations of the two ions of the acid is proportional to the concentration of its undissociated portion, and since the concentration of the negative ion is largely increased by the addition of the neutral salt, the ratio of the concentration of the hydrogen ion to that of the undissociated acid must decrease in the same proportion. In the following experiment, in order to show the difference in the concentrations of the hydrogen ion in the two cases, use is made of the relative effects of the acid, in the absence and presence of its neutral salt, in accelerating the bromate-iodide reaction.

Preparation.--In a liter bottle place $700 \mathrm{cc}$. of distilled water, $20 \mathrm{cc}$. of 0.5 -normal potassium bromate, $20 \mathrm{cc}$. of 0.5 -normal potassium iodide, and ro cc. of starch solution. Place on the lecture table the half-normal solution of chloracetic acid and a normal one of potassium hydroxide; also a $500 \mathrm{cc}$. graduate, 
two I00 cc. graduates, a $200 \mathrm{cc}$. beaker, two $500 \mathrm{cc}$. bottles, a phenolphthalein solution, and some distilled water.

Experiment.-Measure with the large graduate, $350 \mathrm{cc}$. of the bromate-iodide mixture into each of the $500 \mathrm{cc}$. bottles. Measure out $40 \mathrm{cc}$. of chloracetic acid into each of the two roo cc. graduates. To one graduate add $60 \mathrm{cc}$. of water, and mix by stoppering the graduate and shaking. Pour the contents of the other graduate into the beaker, add a few drops of phenolphthalein and about $20 \mathrm{cc}$. of the normal potassium hydroxide solution, adding the last portion slowly with stirring so as to attain closely the neutral point; then add $40 \mathrm{cc}$. more of the chloracetic acid solution and pour the mixture back into the graduate. At the same moment add the contents of the two graduates to the two bottles containing the bromate-iodide mixture, insert the stoppers, and shake vigorously.

Observations.--The blue color appears very quickly in the bottle to which only the chloracetic acid was added, but much more slowly in that containing also the reutral salt.

Remark.-The same principle is further illustrated in connection with the two following experiments.

\section{EXPERIMENT XIV.}

Principle Illustrated.-The extent to which a weak acid is displaced from one of its neutral salts by another acid added to its solution increases with the degree of dissociation of the added acid. For the displacement depends on the fact that the negative ions of the salt and the hydrogen ions of the added acid when brought together unite in part to form undissociated acid, and the quantity of this which forms is, according to the mass action law, greater, the greater the concentration of the hydrogen ions and therefore the greater the dissociation of the added acid.

Preparation.-Dissolve 6 grams of commercial crystallized alloxan and 2 grams of hydroxylamine hydrochlorate in $200 \mathrm{cc}$. of water, heat for an hour on a water-bath, and exactly neutralize the solution of violuric acid so prepared with dilute potassium hydroxide solution, with the help of litmus paper, rinsing off the pieces of paper with water after their immersion in the solution, so as to make the color of the litmus evident.

(About 
$55 \mathrm{cc}$. of a normal solution are required.) Dilute with enough water to make the volume $450 \mathrm{cc}$. Measure six $50 \mathrm{cc}$. portions of this solution into six lecture test-tubes $3 \mathrm{~cm}$. in diameter, placed in a rack provided with a background of white paper. Determine accurately the amount of half normal hydrochloric acid which suffices to decolorize one of these portions to such an extent that it appears of a pale, but distinctly recognizable, pink shade. (Roughly $6 \mathrm{cc}$. will be required. The exact quantity required is designated $\mathrm{M} \mathrm{cc}$. below.) Then entirely decolorize this portion by adding a few more drops of acid. Provide a Io cc. graduated pipette, an additional lecture test-tube, the four half-normal acid solutions used in Expt. VII, a normal potassium hydroxide solution, a phenolphthalein solution, and a ro cc. graduate containing a chloracetate solution made by nearly but not quite neutralizing $\mathrm{M} \mathrm{cc}$. of the half-normal chloracetic acid with normal potassium hydroxide and diluting up to $10 \mathrm{cc}$.

Experiment.-Add potassium hydroxide solution in excess to the colorless violuric acid solution, and remove the tube from the rack. Add by means of the ro cc. graduated pipette to one of the tubes of sodium violurate $\mathrm{Mcc}$. of water to serve as a standard and to the four other tubes $\mathrm{M} \mathrm{cc}$. of the four half-normal acids in the order acetic, chloracetic, sulphuric, and hydrochloric. Pour one-half of the portion to which the chloracetic acid has been added into the empty test-tube; and add to this Io cc. of water; and to the half in the original tube add the Io cc. of chloracetate solution previously prepared after adding to the latter a drop of phenolphthalein solution (to show that it is not alkaline).

Observations. - The colorless violuric acid solution becomes a deep violet when the potassium hydroxide is added. The acetic acid reduces the color to about one-half, and the chloracetic acid to about one-tenth that of the standard; the sulphuric acid gives rise to a pale pink, which, however, is considerably deeper than that produced by the hydrochloric acid. The color of the portion to which both the chloracetic acid and the chloracetate are added is about twice as intense as that of the portion which contains only the chloracetic acid.

Remarks.-The purpose of the addition of potassium hy- 
droxide at the start is to illustrate the facts on which the demonstration of the main principle depends, namely that violuric acid in solution is nearly colorless and that its salt is of a deep violet color. The last part of the experiment on the effect of the neutral salt is a further illustration of the principle here under consideration as well as of that underlying Expt. XIII.

Violuric acid is an acid of about the same strength as acetic acid, the dissociation constants of the two acids being $0.0027^{2}$ and 0.0018 , respectively; consequently, the base divides itself nearly equally between the two acids when, as in the experiment, equivalent quantities of them are simultaneously present. Violuric acid is so very much weaker than sulphuric and hydrochloric acids that it is almost completely displaced by both of them; and, to make evident a difference in their behavior, it is necessary to aroid adding enough hydrochloric acid to cause complete decolorization, and to measure quite accurately the amounts of the two acids added; for ro per cent. excess of the sulphuric acid entirely obscures the difference in their behavior.

EXPERIMENT XV.

Principle Illustrated. - The behavior of indicators used in titrating acids and alkalies is primarily determined by the principles governing the displacement of one acid (or base) from its salt by another. It depends on the relative degree of dissociation of the indicator, which must itself be a very slightly dissociated acid or base, and of the titrated acid (or base.) The behavior of indicators is in some cases also greatly influenced by the phenomenon of hydrolysis. The behavior of di- and tri-basic acids is dependent on the different degrees of dissociation of the acid with respect to its first and second, or first, second, and third hydrogen atoms. More specific explanations follow the experiments described below.

Preparation.-Place $250 \mathrm{cc}$. of distilled water in each of ten lecture jars of $400 \mathrm{cc}$. capacity. Provide the following apparatus and solutions : four $50 \mathrm{cc}$. graduates; several stirring rods with one end flattened at right angles to the rod; two pipettes with rubber

1 The constitutional formula of the acid is

2 Magnanini : Ztschr.phys. Chem., I2, 58.

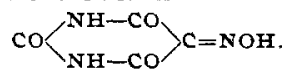


nipples for dropping; half-normal solutions of hydrochloric acid and acetic acid, and normal solutions of potassium hydroxide, ammonium hydroxide, sodium acetate, sodium carbonate, and phosphoric acid (made by diluting I $2 \mathrm{cc}$. of sirupy phosphoric acid of 1.34 specific gravity up to $500 \mathrm{cc}$ ); and solutions of phenolphthalein and methyl orange as ordinarily prepared for volumetric analysis.

Experiment.-I. Add from the dropping pipette, to each of four of the lecture jars containing water 3 drops of the normal potassium hydroxide solution. To the first and third of these jars add five drops of phenolphthalein and to the second and fourth add five drops of methyl orange. Then add from the pipette, drop by drop, half-normal hydrochloric acid to the first and second jars, and half-normal acetic acid to the third and fourth jars with constant stirring, until the indicator becomes decolorized or changes color. In the case of the fourth jar, after showing that the first few drops cause no change in color, add the acetic acid in larger quantities from a graduate until about $40 \mathrm{cc}$. have been added. Then add $25 \mathrm{cc}$. of normal sodium acetate to both the third and fourth jars.

2. To the fifth jar add just $50 \mathrm{cc}$. of half-normal hydrochloric acid and five drops of methyl orange. To the sixth jar add the same amount of the hydrochloric acid and two drops of phenolphthalein. Add to each jar from a $50 \mathrm{cc}$. graduate normal ammonium hydroxide, rapidly until $23-24 \mathrm{cc}$. are added, and then more slowly until the (sharp) end-point is reached in the fifth jar when about $25 \mathrm{cc}$. have been added, and until the solution becomes of a decided red color in the sixth jar, requiring about $40 \mathrm{cc}$. Then add ten drops more of phenolphthalein to the latter.

3. Add to the seventh and eighth jats $25 \mathrm{cc}$. of normal sodium carbonate, and to the former, five drops of methyl orange, and to the latter, five drops of phenolphthalein. Titrate roughly to an end-point with normal hydrochloric acid, adding from a graduate about $50 \mathrm{cc}$. to the seventh jar and $25 \mathrm{cc}$. to the eighth.

4. Add to the ninth and tenth jars $50 \mathrm{cc}$. of normal phosphoric acid and to the former jar five drops of methyl orange and to the latter five drops of phenolphthalein. Titrate roughly to an end- 
point with normal potassium hydroxide, adding from a graduate about $17 \mathrm{cc}$. to the ninth jar and about $34 \mathrm{cc}$. to the tenth.

Observations and Explanations.-I. The end-point is sharp and requires for its attainment the same quantity of acid whether hydrochloric acid with phenolphthalein, hydrochloric acid with methyl orange, or acetic acid with phenolphthalein, is used; but when acetic acid and methyl orange are employed, the change of color is very gradual and requires a much larger quantity of acid. These results are explained as follows: hydrochloric acid is so much stronger (i.e., more dissociated) than either phenolphthalein or methyl orange, and acetic acid is so much stronger than phenolphthalein, that the indicator is completely displaced from its potassium salt by even an inappreciable excess of the acid; on the other hand, since methyl orange is a relatively strong indicator-acid ${ }^{1}$ and acetic acid a relatively weak acid, a large excess of the latter, whereby a considerable concentration of the hydrogen ions results, is necessary in order to displace the indicator completely from its salt. The sodium acetate added at the close of this experiment causes the yellow color of the methyl orange salt to reappear, owing to the great reduction of the dissociation of the acetic acid, whereby it loses its power of displacing the indicator from its salt; this reduction by the sodium acetate is, however, not sufficient to cause any apparent change in the phenolphthalein equilibrium.

2. In titrating hydrochloric acid with ammonium hydroxide, the end-point is sharp when methyl orange is used, while the change to a pink color is very gradual when phenolphthalein is employed. The remarkable behavior of this last indicator is due to the fact that it is such a very weak acid that when the base also is weak, as is the case with ammonium hydroxide, the salt resulting from their union is so readily decomposed by water that an appreciable quantity of it does not form until the ammonium hydroxide is present in considerable excess; on the other hand, methyl orange is so strong an acid that its salts even with weak bases like ammonium hydroxide are not much hydrolyzed. The addition of more phenolphthalein at the end of the exper-

1 Other hypotheses in regard to the behavior of this indicator have been proposed by Kïster (Ztschr.anorg. Chem., I3, I36) and Waddell ( $J$. Phys. Chem., 2, I7I); but these lack experimental verification. 
iment intensifies the red color; for it causes, just as the excess of ammonium hydroxide does, a decrease in the amount of hydrolysis.

3. The results observed in this experiment are indicated in the description of it. They are explained as follows: the acid ion $\mathrm{HCO}_{3}$, which begins to form on the first addition of hydrochloric acid, is less dissociated than either of the two indicator acids and consequently does not displace either of them from its salts. When a little more than one equivalent of acid has been added, however, the acid $\mathrm{H}_{2} \mathrm{CO}_{3}$ begins to form, and since its dissociation is greater than that of phenolphthalein but less than that of methyl orange, it displaces the former, giving an end-point, but does not displace the latter.

4. The results are indicated above. They are readily explained with the help of the following assumptions: The acid $\mathrm{H}_{3} \mathrm{PO}_{4}$ is a stronger acid than either of the indicators; the ion $\mathrm{H}_{2} \mathrm{PO}_{4}$ is a stronger acid than phenolphthalein, but a weaker one than methyl orange; and the ion $\mathrm{HPO}_{4}$ is a weaker acid than either of the two indicators.

\section{EXPERIMFNT XVI.}

Principle Illustrated. - The product of the concentrations of the ions of a salt with which a solution is saturated (the so-called solubility-product) has the same value whether or not other substances are simultaneously present. The presence of a second salt with a common ion therefore reduces the solubility in accordance with this principle; and two different salts, one having in common the positive ion and the other the negative ion of the salt saturating the solution, must have the same effect in reducing the solubility, assuming, as is usually approximately true, that the two salts are equally dissociated.

Preparation of the Experiment.-Prepare $500 \mathrm{cc}$. of a saturated solution of silver acetate by shaking an excess of the solid salt with warm water, cooling, and filtering or decanting. Place $200 \mathrm{cc}$. of this solution in each of two $400 \mathrm{cc}$. lecture jars. Place in graduates $5 \mathrm{cc}$. of fourfold normal solutions of sodium acetate and of silver nitrate; also provide a stirring rod.

Experiment.-Add the measured portion of sodium acetate to 
one jar and that of silver nitrate to the other jar of the silver acetate solution, and stir vigorously for a few moments.

Observations. - A feathery crystalline precipitate of the same character and amount is produced in each jar.

EXPERIMENT XVII.

Principle Illustrated.-The solubility of a salt in water is increased by the addition of a second salt with different ions when there can be produced by metathesis an undissociated substance; and the increase is greater, the greater the amount of this substance formed. Thus, if to saturated solutions of a salt of a partially dissociated acid are added other acids of varying degrees of dissociation, the increase of solubility caused by these will be greater, the greater the degree of their dissociation; for the amount of undissociated acid produced by the metathesis will increase with the increase in the concentration of the hydrogen ions. The formation of the undissociated substance increases the solubility by decreasing the concentration of one of the two ions of the salt saturating the solution, thus making it necessary for more of the salt to dissolve in order to reproduce the original value of the solubility-product.

Preparation.-Treat to grams of salicylic acid with a solution of 3 grams of anhydrous sodium carbonate in $100 \mathrm{cc}$. of water; filter; boil to expel carbon dioxide; add a solution of 9 grams of silver nitrate in $100 \mathrm{cc}$. of water; and wash and dry the precipitate. Place about I gram of it and $45 \mathrm{cc}$. of water in each of four $50 \mathrm{cc}$. wide-mouthed, glass-stoppered bottles. Place in each of four lecture jars Io cc. of hydrochloric acid of I.I2 sp. gr. and $250 \mathrm{cc}$. of distilled water. Piace on the lecture table also the half-normal solutions of sulphuric, chloracetic, and acetic acids used in Expt. VII; a 5 cc. graduated pipette ; a stirring rod; four 25 or $50 \mathrm{cc}$. graduates with a funnel containing a ribbed filter resting in the top of each.

Experiment.-Add, with the help of the graduated pipette, 5 cc. of the three half-normal acids to three of the bottles containing the silver salicylate. Shake all four bottles vigorously for half a minute and pour their contents on to the four ribbed filters. Allow $25 \mathrm{cc}$. to collect in each graduate and pour the four 
$25 \mathrm{cc}$. portions into the four jars of dilute hydrochloric acid in the order, water, acetic acid, chloracetic acid, and sulphuric acid.

Observations. - The jars to which the water solution and the acetic acid solution are added show a very slight and nearly equal turbidity. The turbidity is much greater in the case of the chloracetic acid, and again much greater in the case of the sulphuric acid.

\section{ON THE PREPARATION OF TRIPHENYLCHLORMETHANE.}

By M. GOMBERG.

Received October 4, 1900 .

$\mathrm{T}$ is generally stated in smaller as well as in larger ${ }^{1}$ text-books

on organic chemistry, that when carbon tetrachloride is treated with benzene and aluminum chloride triphenylchlormethane is the principal product of the reaction. This statement is entirely erroneous.

Friedel and Crafts, who were the first to study this reaction, reported ${ }^{2}$ that they obtained in this way tetraphenylmethane. $E$. and $O$. Fischer ${ }^{3}$ were unable to verify this result, but could obtain only triphenylmethane. It is to be presumed that they carried on this reaction in a manner analogous to that followed in the preparation of triphenylmethane from chloroform, $i . e$, the resulting products, after being freed from the chloride of aluminum and the excess of benzene, were subjected to fractional distillation at the high temperature of $200^{\circ}-360^{\circ} \mathrm{C}$. Later, Friedel and $\mathrm{Crafts}^{4}$ found that the results vary according to the manner of procedure. On distilling the crude products at a high temperature they also obtained triphenylmethane and thus confirmed $E_{\text {. }}$ and $O$. Fischer's results. But if the entire mixture as obtained by the action of aluminum chloride and benzene upon carbon tetrachloride is at once treated with water, it furnishes large quantities of triphenylcarbinol. From this they concluded that the reaction results principally in the formation of triphenylchlormethane, $\left(\mathrm{C}_{6} \mathrm{H}_{5}\right)_{3} \mathrm{C} . \mathrm{Cl}$, and this on treatment with water furnishes the corresponding carbinol. They confirmed this

1 Beilstein: Handbuch, II, 287 (3rd edition).

2 Compt. rend., $1453(1877)$.

3 Ann. Chem. (Liebig), 194, 254.

4 Ann.chim. phys., I, 497 (1884). 